\title{
RECURSOS COMPUTACIONAIS AVANÇADOS A MODELAGEM EM 3D AGREGANDO VALOR AOS ATIVOS DA CSN-UPV*
}

\author{
Cleciano Berlando Miranda de Oliveira ${ }^{1}$ \\ Jonathan de Souza Calvelli ${ }^{2}$ \\ Jerri Araújo ${ }^{3}$ \\ Emilio Augusto Ferreira Esquerdo ${ }^{4}$
}

\section{Resumo}

A Engenharia de manutenção em equipamentos siderúrgicos da CSN vem desenvolvendo habilidade e aplicando com ótimos resultados os recursos computacionais avançados com modelagem 3D e simulação numérica através de métodos de elementos finitos. Estes recursos estão se tornado um diferencial na dinâmica dos trabalhos de manutenção com foco em análises de falhas crônicas e respostas rápidas para a solução dos problemas. Caracterizando e estratificando as falhas é possível desenvolver melhorias e defendê-las com maior assertividade e credibilidade. O recurso computacional já está no mercado há tempos e a CSN está na vanguarda na formação de sua equipe própria com um nível avançado e grande potencial para otimização de resultados da engenharia de manutenção de equipamentos (GEM) da CSN-UPV. A missão principal é agregar valor aos ativos, com agilidade e confiabilidade na caracterização de oportunidades de melhorias dos projetos ou restabelecimento funcional. Trilhando um caminho contínuo no que tange a qualidade e assertividade nas soluções de problemas de equipamentos mecânicos, serão apresentados alguns casos de sucesso que estão em desenvolvimento e um projeto de melhoria nos rolos de entrada de um laminador de encruamento, localizado na saída da linha de recozimento contínuo para chapas número um (LRCC\#1). Estes rolos apresentavam fraturas com frequências mensais gerando paradas de mais de 8 horas e, após os estudos e implantação do projeto em 2012, não houve mais registros de paradas por falhas nos rolos. $O$ projeto foi padronizado e multiplicado para os demais rolos da LRCC\#1 e outras linhas com projetos semelhantes.

Palavras-chave: Engenharia de manutenção; Equipamentos siderúrgicos; Modelagem 3D; Métodos de elementos finitos (MEF).

\section{ADVANCED COMPUTING RESOURCES - 3D MODELING ADDING VALUE TO THE COMPANY} ASSETS (CSN-UPV)

\section{Abstract}

The maintenance engineering in steel equipment of "CSN" has been developing skills, and applying them with excellent results in advanced computing resources with $3 \mathrm{D}$ modeling and numerical simulation, using finite element methods. These resources has becoming in a competitive advantage for maintenance works, with focus in the analysis of chronic failures and quick responses for solving problems. Through characterization and stratification of failures, it is possible to develop several improvements and consolidate them with greater assertiveness and credibility. These computational resources are in the market for some time, but CSN is in the "vanguard", once it gave a specialized forming in an advanced level to its own staff, in order to achieve great results in the GEM - equipment maintenance engineering of CSN-UPV. The main goal is adding values to the company assets with great reliability and agility, in order to improve designs or functional recovery of the equipment. Aiming to achieve assertiveness and quality in the troubleshooting of mechanical equipment, will be shown some successful studies and an improvement project of the input rollers at skin-pass mill, located in the output continuous annealing line 1 (LRCC \# 1). These rolls showed fractures with monthly frequency, generating more than 8 hours of loss of production, after studies and project implementation in 2012, there were no more breakdown records related to this rolls. The project has been standardized and applied to the other rolls of LRCC \# 1, as well as for the other lines of CSN with similar projects.

Keywords: Maintenance engineering; Steel equipment; 3D modeling; Finite element methods (FEM).

1 Eng. Mecânico, Mestre em Engenharia Mecânica, Eng. Especialista em Equipamentos, GGOS/GEM, CSN, Volta Redonda, RJ, Brasil.

2 Eng. Mecânico, Pós-Graduado, Eng. de Desenvolvimento Sr., GGOS/GEM, CSN, Volta Redonda, RJ, Brasil.

3 Técnico em Metalurgia, Técnico de Desenvolvimento II, GGOS/GEM, CSN, Volta Redonda, RJ, Brasil.

4 Técnico em Eletromecânica, Técnico, Técnico de Desenvolvimento Especialista, GGGL/GRX, CSN, Volta Redonda, RJ, Brasil. 


\section{INTRODUÇÃO}

A missão da GEM (Gerência de Engenharia de Manutenção) tem como foco a definição de novos paradigmas para os processos de manutenção voltados à redução de custos nos ativos da CSN. Existe uma equipe de especialistas multidisciplinar que desenvolve análises de desempenho de indicadores e equipamentos, análises de falhas críticas e crônicas, melhorias e modificações, suporte na execução de atividade de relevância técnica e restituição funcional dos equipamentos. As análises de falhas começam após as identificações de problemas crônicos nos equipamentos ou baixa performance, impactando negativamente nas metas de produção das áreas e, assim, a equipe GEM inicia todo o processo de análise de falhas utilizando técnicas de prototipagem, modelagem em 3D e análises pelo MEF (Método de Elementos Finitos), discretizando os resultados de montagens e suas interferências, fadigas, concentrações de tensões, regiões de deformações, fator de segurança mínimo, entre outros parâmetros.

\subsection{Recursos Computacionais Utilizados pela GEM}

A GEM utiliza o Autodesk Inventor [1] para a modelagem e simulações simplificadas e, para simulações mais avançadas, conta com o ABAQUS ${ }^{\circledR}$ [2]. Os especialistas efetuam uma discretização das complexidades dos equipamentos com confiáveis análises e testes virtuais. Isso, consequentemente, diminui a necessidade da criação de protótipos físicos e, também, os custos. Nas análises de resistências das peças ou conjuntos mecânicos os softwares automáticos são primordiais, porém, é necessária a conceituação e formulação da teoria da elasticidade [3] para a aplicação e interpretação dos resultados nas análises estáticas e dinâmicas, onde o comportamento de qualquer sistema físico é regido por equações diferenciais ou integrais, como na mecânica dos sólidos deformáveis por exemplo. Com a preparação da equipe nas práticas de modelagem e análise por MEF, iniciada em 2009, foi possível um crescimento significativo da equipe própria reduzindo as necessidades de contratações dessa modalidade de serviços. Na figura 1 representamos graficamente a evolução que a CSN vem consolidando ao longo dos últimos anos e, na sequência, figura 2, ilustramos alguns dos casos mais relevantes desenvolvidos sob a luz dos Recursos Computacionais Avançados.

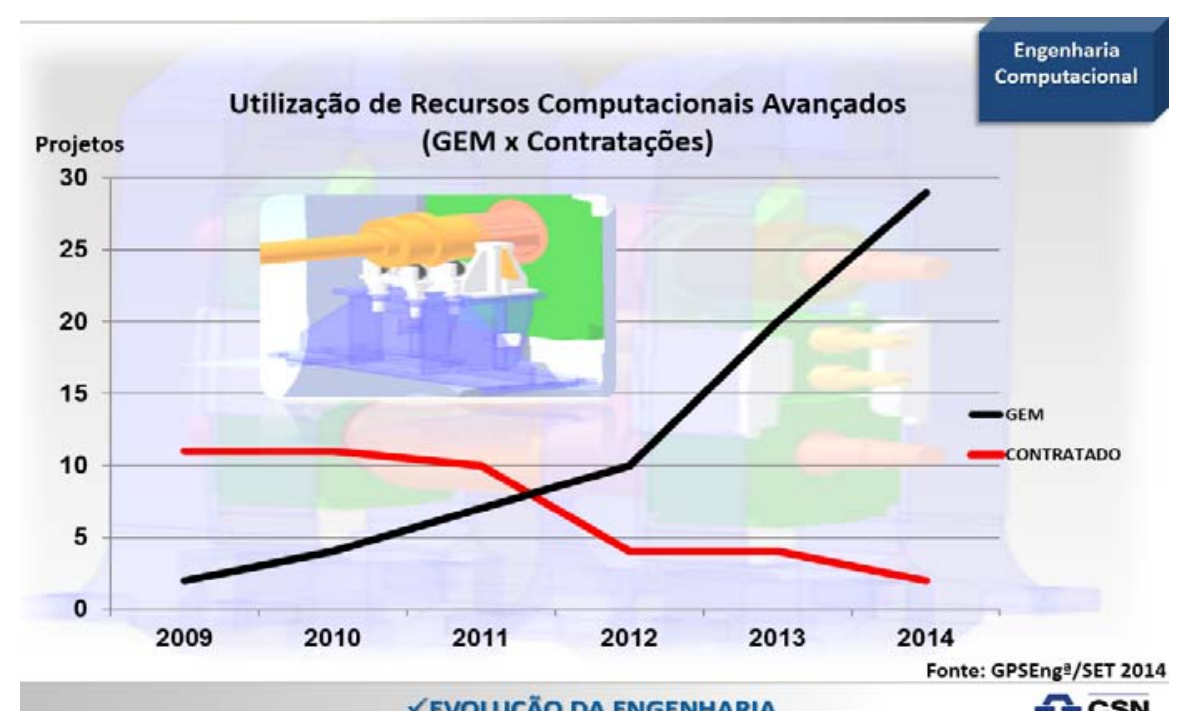

Figura 1. Ilustração da redução de contratações de estudos de MEF e absorção por equipe própria. 


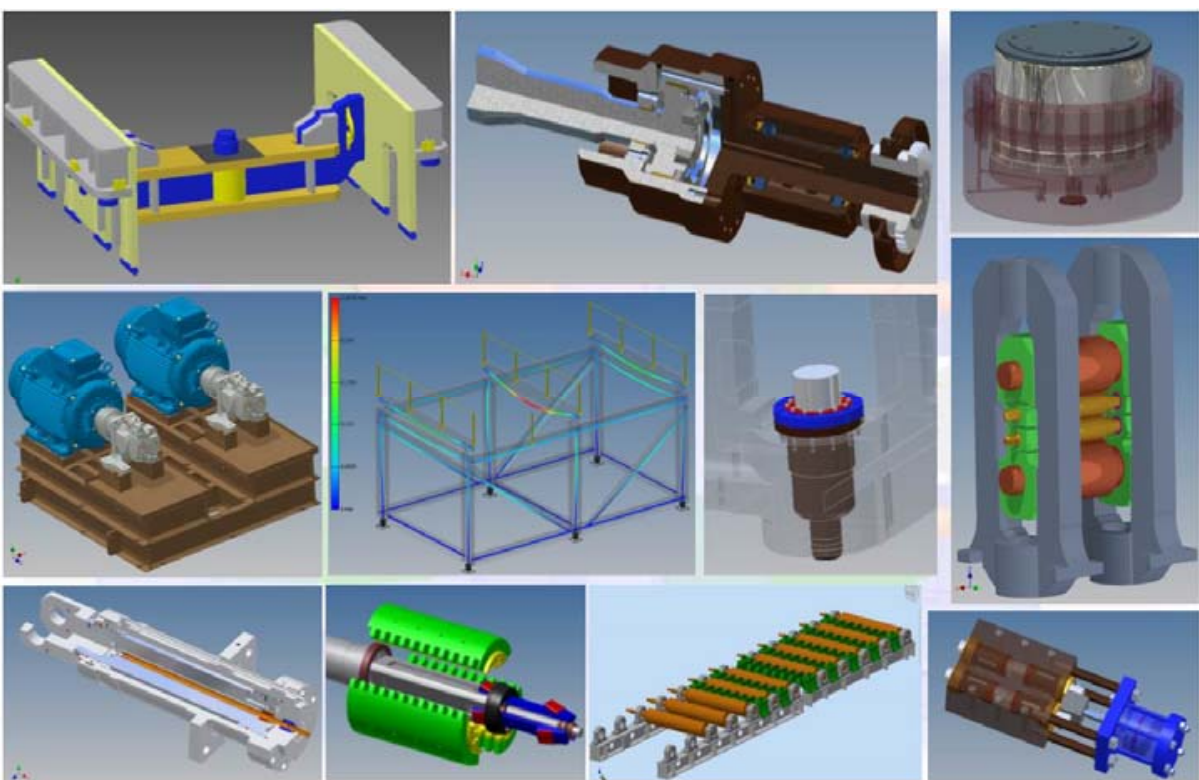

Figura 2. llustração de trabalhos desenvolvidos com modelagem em $3 \mathrm{D}$, estudos de MEF e funcionalidades

\subsection{Evolução do Desenvolvimento do Método de Elementos Finitos[4]}

Conhecer o desenvolvimento deste método nos permite melhor valorizá-lo, sob suas múltiplas facetas. Por isso, segue um relato conciso da evolução desse desenvolvimento, sem detalhamento que possa desviar o leitor do objetivo principal de compreender este método.

O MEF foi iniciado pelos engenheiros aeronáuticos Turner, Argyris e Associados, na segunda metade da década de 50 , como resultado da evolução da Análise Matricial de Estruturas e do advento do computador. A abordagem foi intuitiva, através do Princípio dos Deslocamentos Virtuais, sem o conhecimento de critérios de convergência. $E$ dado à exploração espacial, este método teve rápido desenvolvimento e reconhecimento, o que contribuiu para a elaboração de bem sucedidos projetos de espaçonaves, mísseis e cápsulas espaciais, com consequentes aplicações em diversas outras áreas da Engenharia.

Na primeira metade da década de 60, identificou-se que o MEF pôde ser entendido como um caso particular do Método de Rayleigh-Ritz e como tal formulado a partir de funcionais. Isso estendeu a aplicabilidade deste método a problemas não estruturais, como de fluídos, meios porosos, termodinâmica e eletromagnetismo, e conduziu ao estabelecimento de critérios de convergência. No final da mesma década, comprovou-se que o presente método pode ser formulado também a partir de equações diferenciais com condições de contorno, como um caso particular do Método de Galerkin. Com isso, o MEF passou a não requerer a existência de um funcional, com maior ampliação de sua aplicabilidade.

$\mathrm{Na}$ década de 70 , identificou-se que o presente método pode ser compreendido como um caso particular dos métodos de resíduos ponderados em geral e não apenas do Método de Galerkin, com consequente nova ampliação de sua aplicabilidade e de suas vertentes de formulação. Ainda nessa década, foram disponibilizados programas automáticos para a análise de uma ampla gama de sistemas físicos, com a incorporação de facilidades de geração de modelos discretos e com a inclusão de eficientes algoritmos de resolução dos sistemas de equações algébricas e dos problemas de autovalor decorrentes desses modelos. 
$\mathrm{Na}$ década de 80 , foram discretizados de forma satisfatória modelos matemáticos com mais de um tipo de energia e que ficam inconsistentes no caso de nulidade de um dos tipos de energia envolvidos. É o que se chama problemas de restrições internas, como em sólidos e fluídos quase-incompressíveis, e em placas e cascas muito finas analisadas com as hipóteses de Reissner-Mindlin. E ainda nessa década, o presente método passou a ser disponibilizado em microcomputadores, o que o tornou mais acessível.

Na década de 90 , dado à ampla disponibilidade de microcomputadores e programas comerciais de baixo custo, este método se popularizou com eficientes ferramentas de pré e de pós-processamento, o que facilitou o seu uso em modelos com expressivos números de graus de liberdade.

Atualmente, o MEF está consolidado, tem suas bases matemáticas perfeitamente esclarecidas e é rotineiramente utilizado em projetos de engenharia. Suas programações automáticas estão cada vez mais eficientes, entre as quais merecem citação: ANSYS ${ }^{\circledR}$, NASTRAN $^{\circledR}, \operatorname{COSMOS}^{\circledR}$, ABAQUS $^{\circledR}$, ALGOR $^{\circledR}$, SAP $^{\circledR}$ e ADINA $^{\circledR}$

\section{MELHORIAS NOS ROLOS DE ENTRADA DO LE\#5 COM UTILIZAÇÃO DE MODELAGEM EM 3D E MEF}

$\mathrm{Na}$ apresentação de um caso de sucesso, com a utilização das metodologias de análise de falhas e os recursos computacionais na prototipagem digital, modelagem em 3D e Métodos de Elementos Finitos, foi possível elevar o patamar de disponibilidade do Laminador de Encruamento $\mathrm{n}^{\circ} 5$ da Linha de Recozimento Contínuo de Chapas da CSN. Na sequência é apresentado um preâmbulo do funcional da Linha, as metodologias e estratificação dos resultados antes e depois da aplicação das melhorias nos rolos.

\subsection{Fluxo do Processo Produtivo do LRCC\#1 [5]}

A Linha de Recozimento Contínuo para Chapas $n^{\circ} 1$ (LRCC\#1), inicia seu processo contínuo através de duas desenroladeiras de bobinas de chapas fazendo com que elas iniciem sua trajetória através dos rolos, também chamado de "caminho da tira". Durante o percurso a tira passa por vários equipamentos com velocidades distintas e o que possibilita estas variações de velocidades e até paradas pontuais, como por exemplo, na hora de fazer as soldas de emenda entre bobinas para tornar o processo contínuo, é a torre de entrada (torre de acumulação) e o loop car de saída, ou seja, enquanto o final da bobina está parado na máquina de solda fazendo a fusão com o começo de outra bobina, a tira continua passando em velocidade constante nos fornos e no laminador de encruamento chegando até as enroladeiras, onde se retiram as bobinas acabadas prontas para os clientes finais ou internos. A tensão na tira é controlada pelos rolos tensores aplicados em pontos funcionais da linha e, inclusive na entra e saída do LE\#5, onde estes foram alvo do estudo e desenvolvimento das melhorias mediante uma necessidade de eliminar as várias falhas crônicas na estrutura dos rolos. 


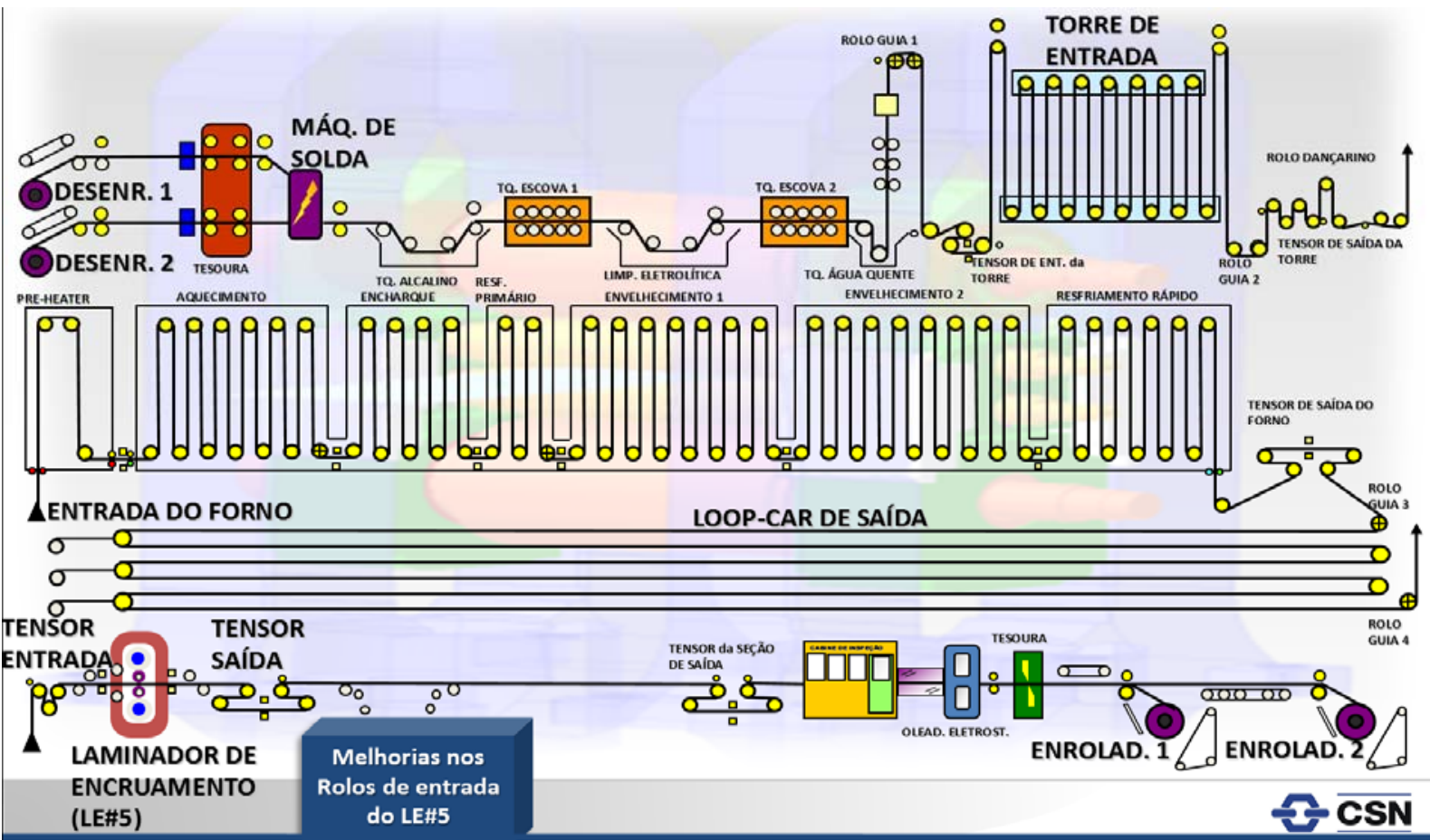

Figura 3. Fluxo do processo produtivo da LRCC\#1 - Visão geral do processo.

\subsection{Condições Operacionais e Produtivas da LRCC\#1}

A LRCC\#1 iniciou sua operação em 1988 e está ligada à Gerência Geral de Galvanizados e Laminados a Frio (GGGL). Atualmente produz 1400 toneladas por dia de produtos para entrega direta e para beneficiamento em outras unidades internas da CSN. O laminador de encruamento $n^{\circ} 5$ sofreu uma modernização em 2001 de todo sistema de automação (Troca do sistema de controle de aperto ALSTOM), viabilizando a possibilidade de aumento do estiramento da tira, porém não foi feito na época um redimensionamento mecânico dos componentes periféricos, onde se inclui os acionamentos dos rolos tensores e os próprios rolos. $\mathrm{O}$ alongamento máximo do projeto inicial era de $3,2 \%$ com uma tração máxima de 4875 kgf e passou para 6\% com tração máxima de 9010 kgf após a modernização.

Tabela 1. Dados operacionais da LRCC\#1

\begin{tabular}{l|l}
\hline Parâmetros & Dados \\
\hline Projeto Original de 1988 & Nippon Steel \\
\hline Capacidade de produção projeto & 460.000 t/ano \\
\hline Tração máxima na tira - Projeto inicial & $4875 \mathrm{kgf}$ \\
\hline Capacidade de produção atual & 500.000 t/ano \\
\hline Tração máxima na tira - Após “Revamp" & $9010 \mathrm{kgf}$ \\
\hline Quantidade de Rolos em toda Linha & 521 (Caminho da Tira) \\
\hline Produto & Bobina a Frio Recozida (BF) \\
\hline "REVAMP" da Automação do LE\#5 - Sem mecânica & 2001 \\
\hline
\end{tabular}




\subsection{Estratificação da Indisponibilidade da LRCC\#1}

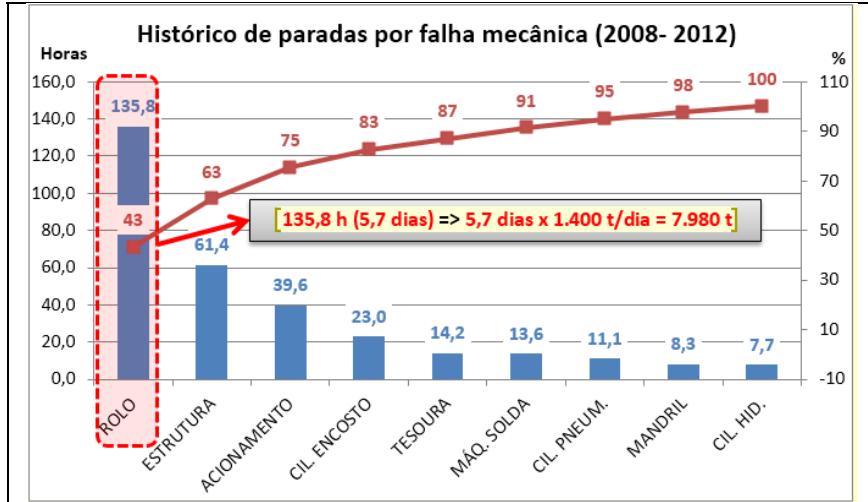

Figura 4.a - Levantamento das ocorrências de falhas mecânicas

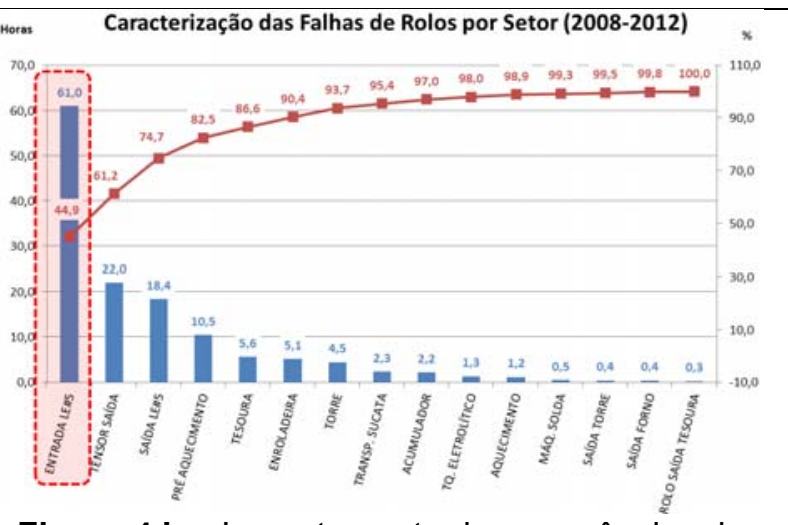

Figura 4.b - Levantamento das ocorrências de falhas dos 521 rolos

Figura 4. Levantamento das ocorrências de falhas para justificar o trabalho de melhorias nos rolos da LRCC\#1 - Fonte: SIGMA.

Com a estratificação da disponibilidade da linha verificou-se e se justificou a necessidade do desenvolvimento do trabalho de melhorias nos índices de falhas dos rolos, onde ficou definido que o trabalho seria desenvolvido nos tensores de entrada do LE\#5, por ter o maior impacto em paradas da linha relacionado a falhas em rolos.

\subsection{Identificação do Problema}

Durante os estudos com levantamento de campo encontrou-se vários rolos com fraturas nas soldas das juntas entre mangas (eixos) e espelhos (discos de chapas que unem os eixos com a carcaça calandrada dos rolos).

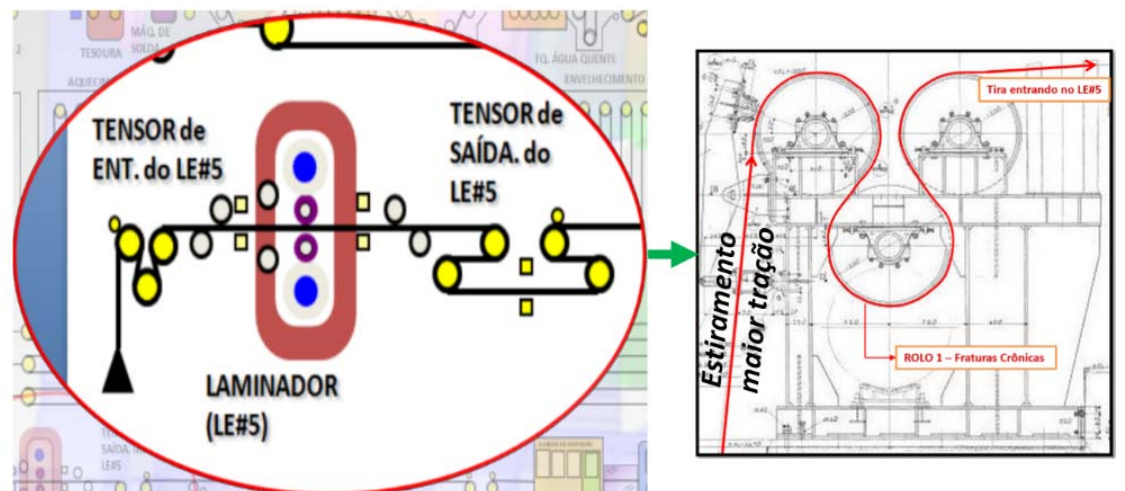

Figura 5a. Ilustração do LE\#5, Tensores de Entrada e Saída - Caminho da Tira com estiramento acima do previsto no projeto mecânico original em função da modernização no controle de força.

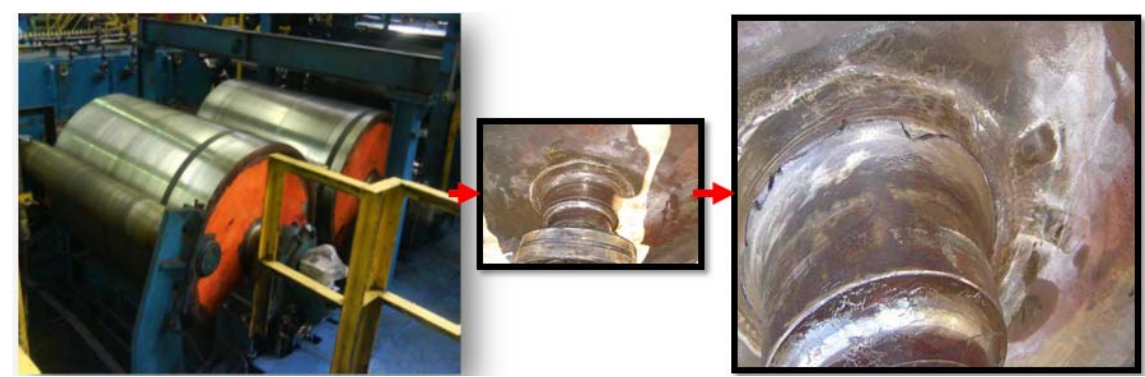

Figura 5b. llustração da falha crônica na junta soldada entre a manga do eixo e o espelho. 


\subsection{Análise da Falha}

Todos os rolos tensores da entrada e saída do LE\#5 são motorizados e controlados pelo sistema da automação que sofreu modernização (Revamp 2001), onde este sistema controla o estiramento da tira que por sua vez exerce solicitações mecânicas acima do previsto no projeto original, conforme pode ser observado na tabela 1. As figuras 4 e 5 identificam o rolo $n^{\circ} 1$ do tensor de entrada com uma fratura que tem uma taxa de frequência elevada, em intervalos de aproximadamente três meses, porém os outros rolos dos tensores de entrada e saída do laminador sofrem o mesmo tipo de falha, sendo assim o projeto piloto foi todo desenvolvido para o rolo $\mathrm{n}^{0} 1 \mathrm{com}$ uma projeção e previsão de verba para aplicação em todos os 06 rolos.

Durante os estudos aplicando as metodologias de análise de falhas [6], foram definidas as possíveis causas que levavam os rolos às fraturas crônicas. Assim se fez um delineamento para o processo de engenharia investigativa que pudesse subsidiar as ações necessárias para mitigar todos os fatores que contribuíam para as falhas. Os fatores considerados de maior relevância pela equipe, para a solução do problema foram: Inspeções do rolo fraturado; Análises do projeto com técnicas de modelagem em 3D e MEF aplicando melhorias.

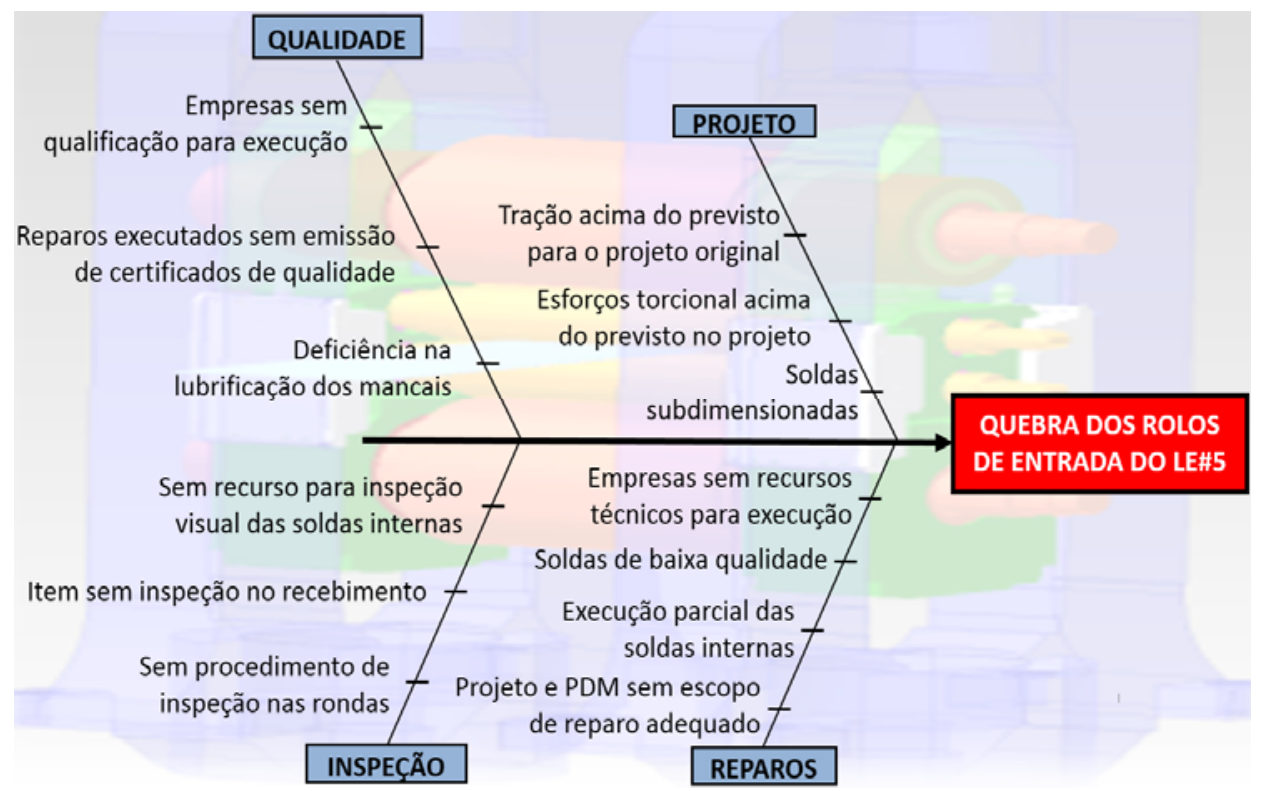

Figura 6. Diagrama de Causa e Efeito para avaliação das quebras dos rolos.

\subsubsection{Inspeção do rolo fraturado}

Através do recurso de videoscopia foi possível identificar fratura em soldas internas e até mesmo falta de solda contínua nestas juntas.

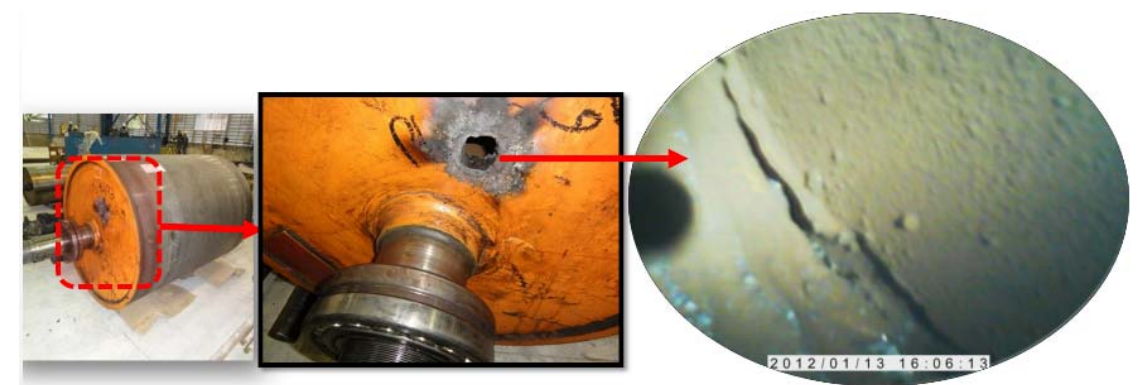

Figura 7. Inspeção visual por videoscopia (Boroscópio) - Recurso próprio da GEM. 


\subsubsection{Análises do Projeto Original com Técnicas de Modelagem em 3D e MEF}

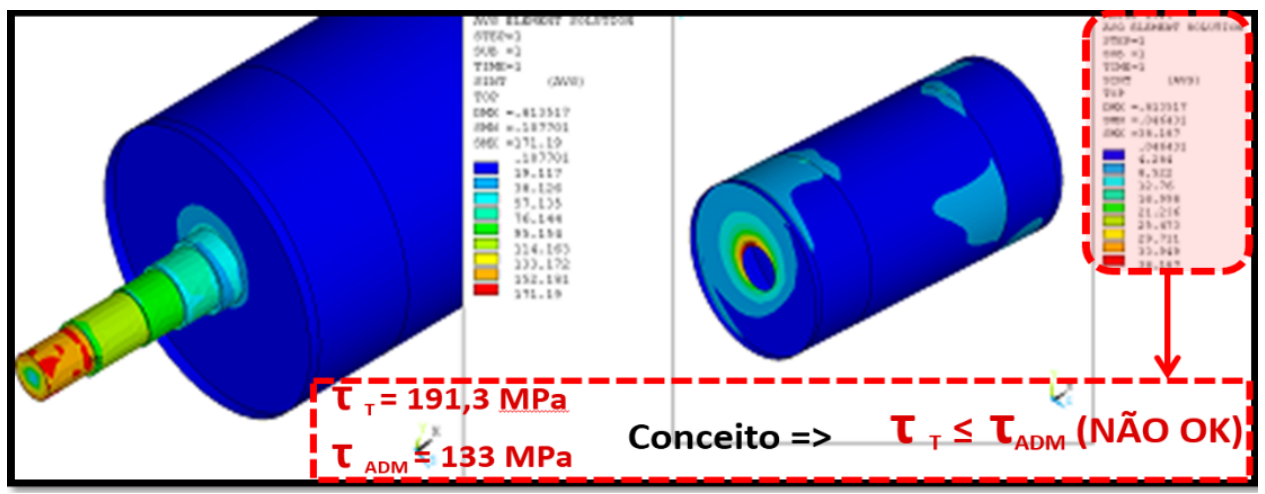

Figura 8. Análise do rolo pelo Método de Elementos Finitos (MEF) - Projeto Original, Tensão de Trabalho acima da Tensão admissível

\subsection{Desenvolvimento de Melhorias com Técnicas de Modelagem 3D e MEF}

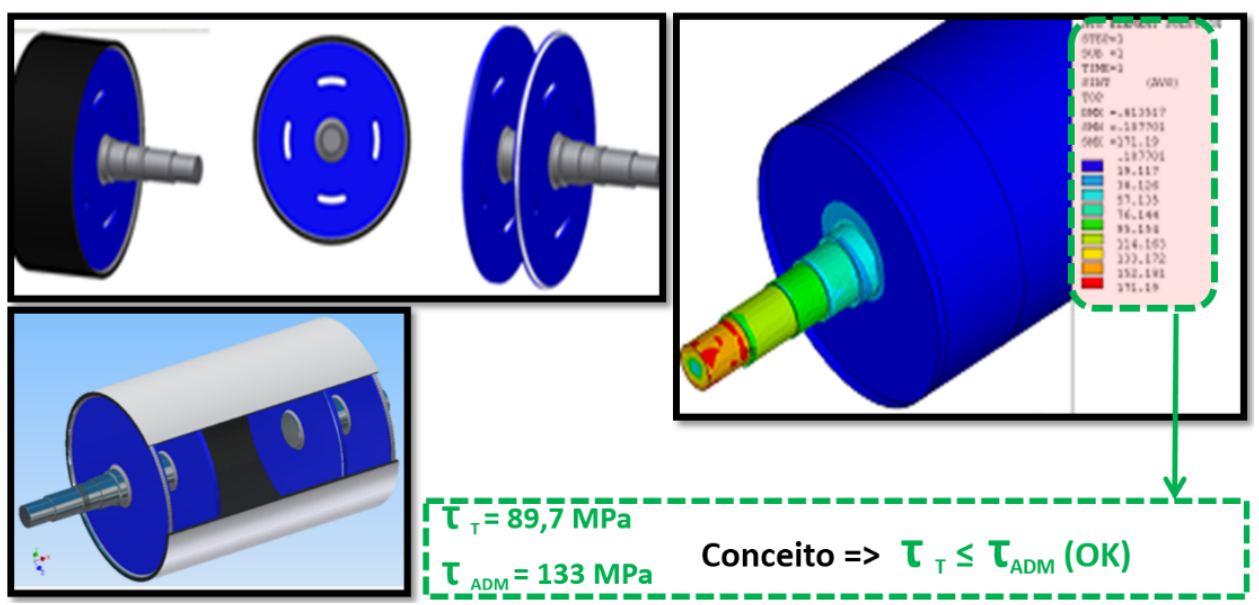

Figura 9. Análise do rolo pelo Método de Elementos Finitos (MEF) - Projeto com Melhorias, Tensão de Trabalho abaixo da Tensão admissível.

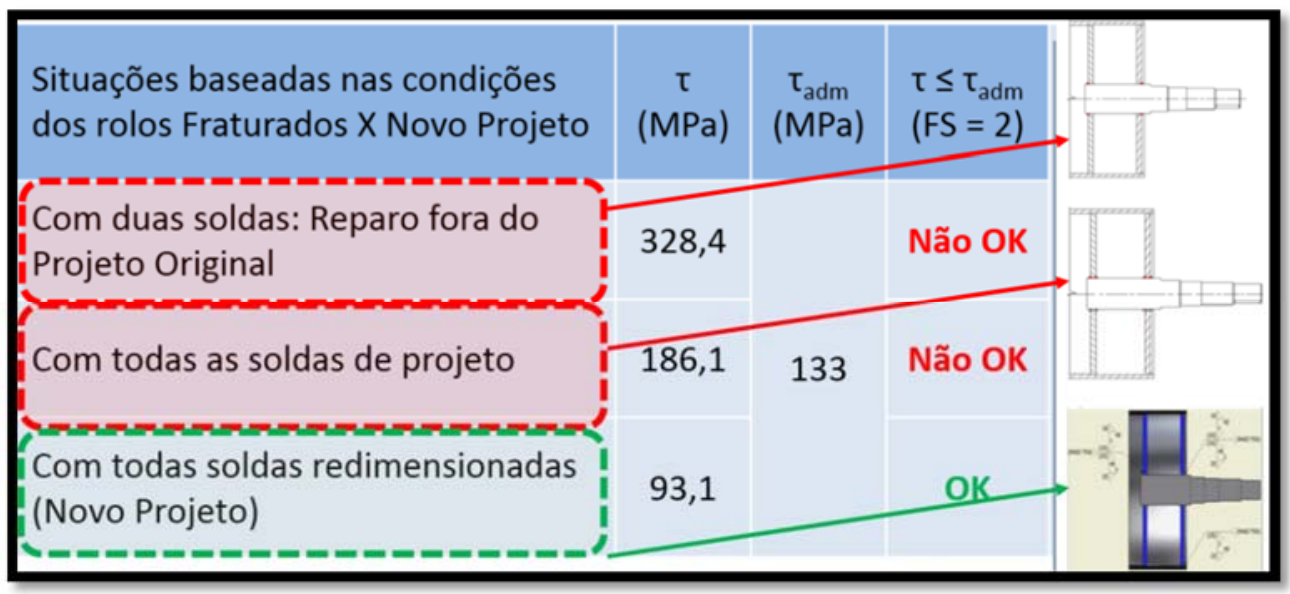

Figura 10. Validação Analítica da Simulação Computacional conforme Norma (ASME Sec. VIII Div 2 para aço ASTM A-36). 


\section{VERIFICAÇÃO DOS RESULTADOS}

Conforme demonstrado nos gráficos das figuras 11 e 12, pode-se confirmar que, após a implantação dos rolos com as melhorias previstas no projeto, a LRCC\#1 superou as expectativas da Diretoria quanto a eliminação das falhas nos rolos e consequentemente aumentou o índice de disponibilidade da linha como um todo. Após 2014 a área continuou fazendo a replicação das melhorias para os outros rolos e até março/2016 não se teve nenhuma falha nos rolos tensores e nem em rolos similares que sofreram as adaptações.

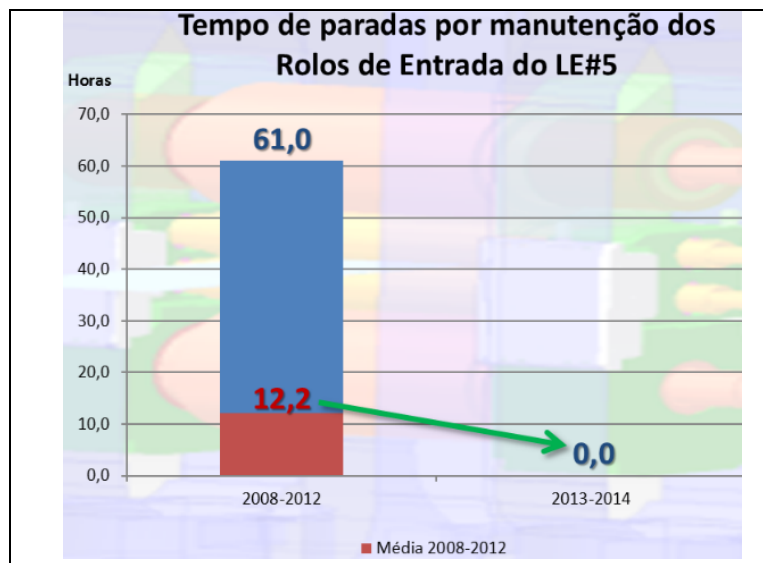

Figura 11a. Verificação do índice de falha dos rolos após implantação das melhorias

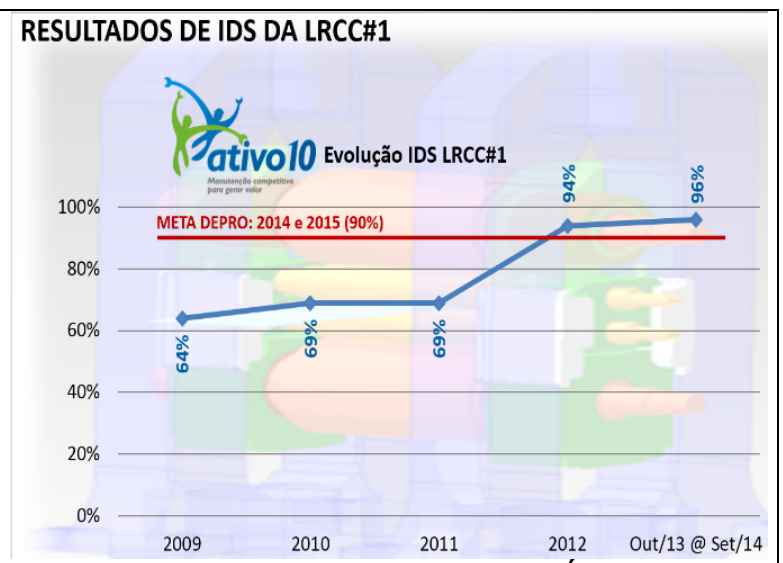

Figura 11b. Resultados de IDS (Índice de Sistematização da Manutenção) - Meta: $88 \%$

Figura 11. Levantamentos dos resultados e confirmação da melhora de performance da LRCC\#1 superando as metas da Diretoria de Produção da CSN (DEPRO) - 2008 até 2014.

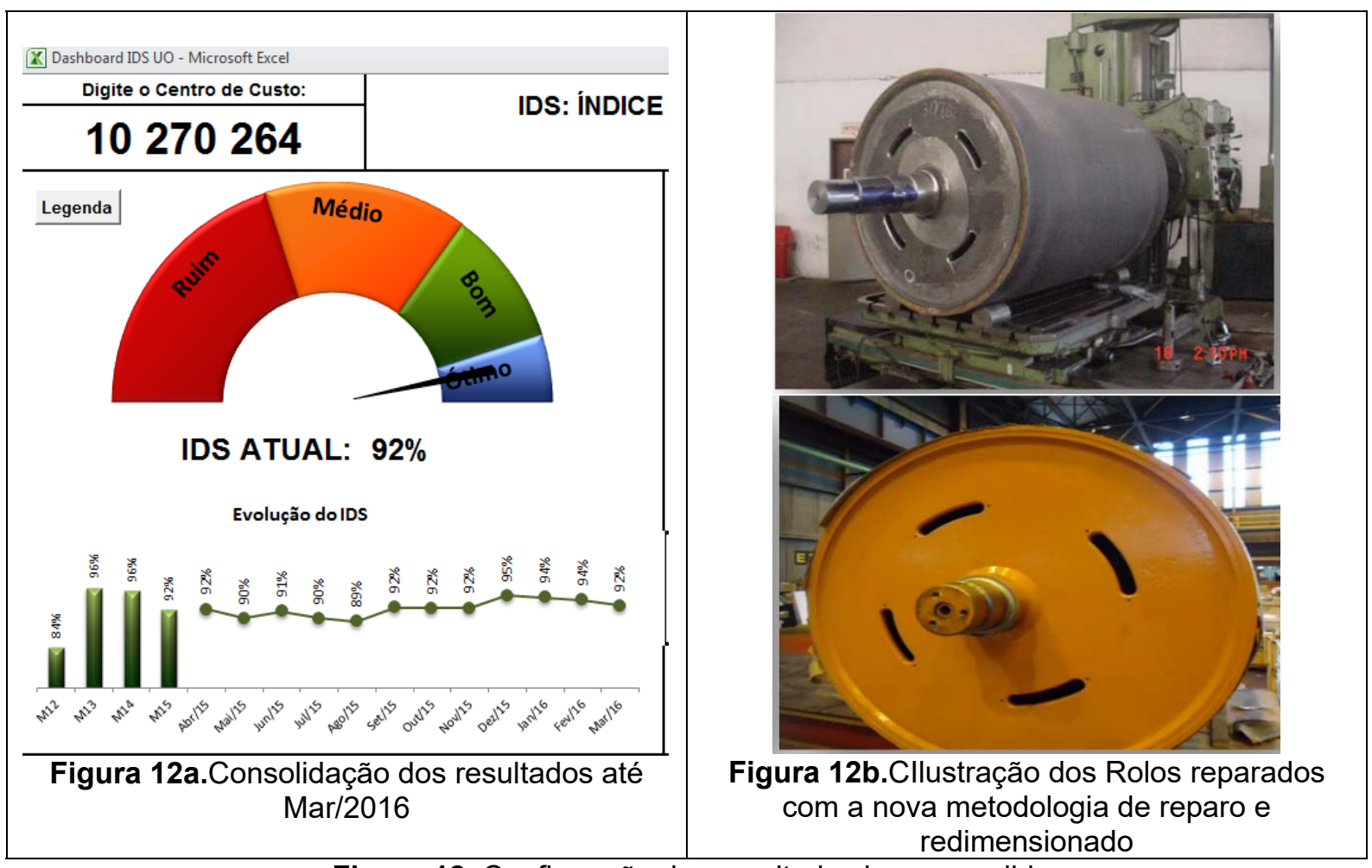

Figura 12. Confirmação dos resultados bem sucedidos. 


\section{CONCLUSÃO}

O recurso computacional avançado com modelagem 3D e simulação em MEF são as formas mais modernas e com possibilidades de ganhos tanto na avaliação de um equipamento quanto na automatização para avaliações análogas. Foi possível comprovar o poder dos recursos computacionais avançados nesse trabalho de melhorias dos rolos de uma linha contínua da CSN e também em outros projetos de grande relevância para garantir a operacionalidade dos ativos do Grupo com:

\section{a) Diferencial na dinâmica de análise de falhas crônicas;}

\section{b) Respostas rápidas para solução de problemas;}

\section{c) Caracterização e estratificação das falhas com maior assertividade.}

\section{Agradecimentos}

A CSN pelo investimento sistêmico na evolução da equipe e a todos os participantes desse trabalho, que continua superando os resultados esperados.

\section{REFERÊNCIAS}

1 Autodesk INVENTOR ${ }^{\circledR}$ Versão 2015 (atualizada anualmente).

2 ABAQUS ${ }^{\circledR}$ Versão 2014.

3 Timoshenko, S.P.; Goodier, J.N., Teoria da Elasticidade, $3^{a}$ Edição - Coyright by Editora GUANABARA DOIS S.A., Rio de Janeiro, 1980.

4 SORIANO, Humberto Lima, Elementos Finitos - Formulação e Aplicação na Estática e Dinâmica das Estruturas. Rio de Janeiro: Editora Ciência Moderna Ltda., 2009.

5 LRCC\#1, Linha de Recozimento Contínuo para Chapas, Companhia Siderúrgica Nacional.

6 Campos, Vicente Falconi, Gerenciamento da Rotina do Trabalho do Dia-a-Dia, $6^{\mathrm{a}}$ Edição - Editora de Desenvolvimento Gerencial, Belo Horizonte, 1998. 